\title{
TANGENTIAL ACCELERATION OF HELIUM PLASMA IN LABORATORY CURRENT SHEETS
}

\author{
N.P. Kyrie ${ }^{1}$
}

\begin{abstract}
Results are presented from studies of the process of plasma acceleration along the current sheet width under different experimental conditions. The behavior of the neutral component is included in the analysis.
\end{abstract}

\section{Introduction}

Laboratory-produced current sheets are particularly well suited for studies of flare processes in plasma, including solar flares and substorms in magnetospheres of Earth and other planets (Syrovatskii 1981; Priest \& Forbes 2000). Current sheets (CSs) may form in magnetic fields with $\mathbf{X}$-type singular lines (Frank 2010; Frank 2012). A current sheet (CS) is a magnetoplasma structure characterized by two markedly different cross-sectional dimensions, namely, thickness and width. Generally, the sheet thickness is smaller than the sheet width by a factor of $5-10$.

This paper is devoted to studies of the process of plasma acceleration along the CS width, i.e. the tangential acceleration of the plasma.

\section{Experimental device}

Investigations were carried out with the CS-3D device, Figure 1. A quasi-steady magnetic field with an $\mathbf{X}$ line on the $z$-axis $\mathbf{B}=\left\{h y ; h x ; B_{z}^{0}\right\}$ is formed by currents flowing in coils and straight conductors; which are situated around a 18-cm-diameter 100 -cm-long quartz vacuum chamber so that the $\mathbf{X}$ line coincides with the chamber axis. In the described experiments, the gradient of the magnetic field in the $(\mathrm{x}, \mathrm{y})$ plane was $h=0.5 \mathrm{kG} / \mathrm{cm}$, the longitudinal magnetic field

This work is supported by the Russian Foundation for Basic Research (project No. 12-02-00553a) and the RAS Program of Fundamental Research N15 "Plasma Processes in Space and Laboratory".

1 A.M. Prokhorov Institute of General Physics of the Russian Academy of Sciences, 38 Vavilov Str., Moscow 119991, Russia; e-mail: kyrie@fpl.gpi.ru 
was $B_{z}^{0}=0-3 \mathrm{kG}$, and the vacuum chamber was filled with helium up to a pressure 100-320 mTorr. The initial plasma was generated in magnetic field $\mathbf{B}$ using an auxiliary $\Theta$-discharge with strong preliminary ionization. In the prepared plasma, the current $J_{z}$ is generated by applying a pulse voltage of $U_{z}=15 \mathrm{kV}$ between two end electrodes located at a distance of $\Delta \mathrm{z}=60 \mathrm{~cm}$ from each other. Electric current $J_{z}$ was almost sinusoidal with amplitude $70 \mathrm{kA}$ and half-period $6 \mu \mathrm{s}$. For more details, see (Frank 2012).

\section{Spectroscopic measurements}

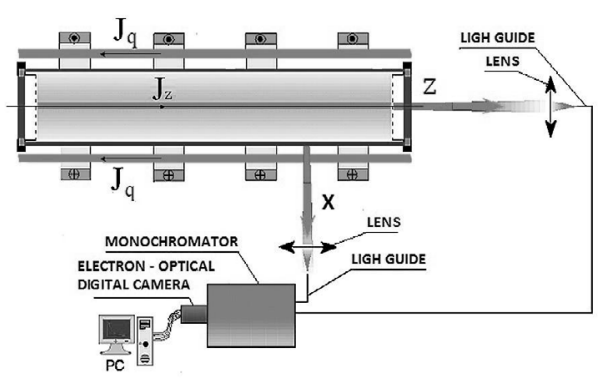

Fig. 1. Schematic of the CS-3D device (lateral view) and spectroscopic equipment.
The energy of directed motion, temperatures of atoms and ions, and electron density in different regions inside the sheet were derived from measurements of broadening of the spectral lines (SLs) of helium atoms and ions: He I $4471 \AA\left(4^{3} \mathrm{D}-2^{3} \mathrm{P}\right)$, He I $5876 \AA$ $\left(3^{3} \mathrm{D}-2^{3} \mathrm{P}\right)$, He II $3203 \AA \quad((\mathrm{n}=5)-$ $(\mathrm{n}=3))$, He II $4686 \AA((\mathrm{n}=4)-(\mathrm{n}=3))$. Both the Doppler and Stark effects contribute to the width of the SLs, but constants for these effects are different. This distinction allowed us to determine the velocities of atoms and ions as well as the electron density in different regions of the sheet.

It should be noted that the structure directed plasma motions in CSs is usually rather complicated: plasma moves both towards the observer and away from him, and the plasma velocity varies along the line of sight. As a result, instead of shifting the SLs undergo additional broadening as compared to their thermal widths. To distinguish between the velocities of directed and thermal motion, measurements were carried out in two mutually perpendicular directions, Figure 1, see also (Kyrie et al. 2010).

\section{Main experimental results}

Figure 2 compares the profiles of the ionic line He II $4686 \AA$ observed in the $x$ - and $z$-directions in the $2 \mathrm{D}$ magnetic configuration $\left(B_{z}=0\right)$. The line width observed in the $x$-direction is nearly 3 times larger than the width in the $z$-direction. Similar results were observed for the He II $3203 \AA$ line.

Analysis of the profiles of both ionic lines showed that they can be approximated by a Voigt function, i.e. by convolution of a Gaussian with Lorentzian. The Gaussian shape fits the Doppler and instrumental broadening, whereas the Lorenz (dispersion) profile is attributed to the Stark effect which under our experimental conditions may be associated with the electron-impact broadening mechanism. 


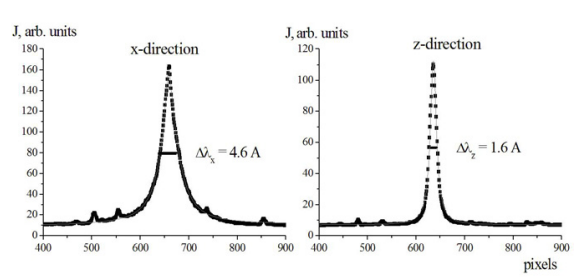

Fig. 2. Profiles of the He II $4686 \AA$ spectral line emitted from current sheet plasma in $x$ - and $z$-directions. Experimental conditions: $h=0.5 \mathrm{kG} / \mathrm{cm}$, $B_{z}^{0}=0, p=320$ mTorr, $J_{z}=70 \mathrm{kA}$, $t=(4.3 \pm 0.4) \mu \mathrm{s} .100$ pixels $=12.5 \AA$.

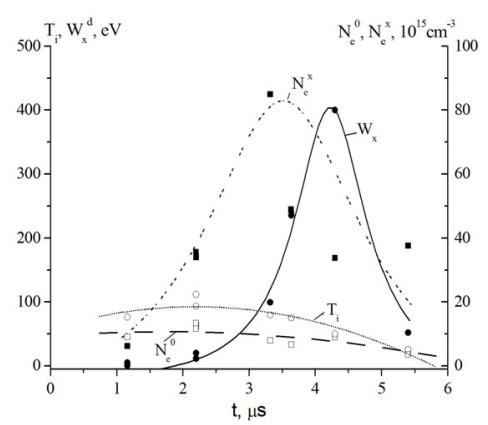

Fig. 3. Time behavior of the electron density $N_{e}^{0}$ and $N_{e}^{x}$, ion temperature $T_{i}$, and energy of directed motion $W_{x}$ measured in 2D magnetic configuration. Experimental error in determining $N_{e}^{0}, N_{e}^{x}$ and $T_{i}$ is $10 \%$; error in determining $W_{x}$ is $20 \%$. Experimental conditions are the same as in Figure 2.

From broadening profiles like those in Figure 2, we determined the average energies of thermal and directed motion of helium ions in the $x$-and $z$-directions and the electron density in different regions of the plasma sheet, Figure 3 (Ackermann et al. 1985; Pittman \& Fleurier 1986).

Early in the discharge, at $t=1.2-2.4 \mu \mathrm{s}$, when the electron density in the central region of the CS was $N_{e}^{0} \approx 10^{16} \mathrm{~cm}^{-3}$ and the ion temperature was $T_{i} \approx$ $90 \mathrm{eV}$, the ion energies measured in the $x$ - and $z$-directions were almost equal in value and corresponded to the thermal energy.

Then the energy of directed motion $W_{x}$ began to grow rapidly and reached its maximum $W_{x} \approx 400 \mathrm{eV}$ at $t=(4.3 \pm 0.4) \mu$ s which was about 8 times greater than the thermal energy in the central region of the sheet. In the meantime, the electron density near side edges of the sheet increased to $N_{e}^{x}=3.4 \cdot 10^{16} \mathrm{~cm}^{-3}$, while the density in the central region decreased somewhat to $N_{e}^{0}=0.9 \cdot 10^{16} \mathrm{~cm}^{-3}$.

In $3 \mathrm{D}$ case $\left(B_{z}^{0}=2.9 \mathrm{kG}\right)$, no evidence was found for the existence of directed plasma motion in the $x$-direction. Characteristically, the electron density and ion temperature in the central region changed but insignificantly, and the density values measured in the $x$ - and $z$-directions turned out approximately equal, Table 1 .

The characteristic features of the CS in 2D field became all the more evident, when the initial helium pressure was decreased to $p=100$ mTorr, Table 1 . At the time $t=(3.0 \pm 0.4) \mu \mathrm{s}$, the average ion energy of directed motion reached $W_{x} \approx 1300 \mathrm{eV}$ which value is higher than the ion temperature in the central region by factor of 30 . Under these conditions, the central electron density was somewhat lower, $N_{e}^{0}=0.3 \cdot 10^{16} \mathrm{~cm}^{-3}$, whereas the density near side edges increased 
substantially $N_{e}^{x} \approx(1.8 \pm 0.2) \cdot 10^{17} \mathrm{~cm}^{-3}$. By contrast, in $3 \mathrm{D}$ case, both $W_{x}$ and $N_{e}^{x}$ decreased, Table 1 .

We found that the dipole-allowed line He I $4471 \AA\left(4^{3} \mathrm{D}-2^{3} \mathrm{P}\right)$ was accompanied by the dipole-forbidden line He I $4470 \AA\left(4^{3} \mathrm{~F}-2^{3} \mathrm{P}\right)$ which showed up in the spectrum. The intensity of the forbidden lines measured in the $x$-direction could at times be equal to the intensity of the allowed lines. Such equality indicates that the electron density at the sheet edges reaches $N_{e}^{x} \geq 10^{17} \mathrm{~cm}^{-3}$ (Barnard et al. 1969). Based on a comparative analysis of the profiles of He I $4471 \AA$ and He I $5876 \AA$ lines measured in two directions, we revealed that helium atoms also accelerate along the sheet surface to superthermal velocities, probably, in consequence of charge exchange of fast helium ions with neutrals.

Table 1. Dependence of plasma parameters on the initial gas pressure and magnetic strength $B_{z}^{0}\left(h=0.5 \mathrm{kG} / \mathrm{cm}, J_{z}^{\max }=70 \mathrm{kA}, t=4.3 / 3.0 \mu \mathrm{s}\right)$.

\begin{tabular}{|c|c|c|c|c|}
\hline & \multicolumn{2}{|c|}{$\mathbf{p}=\mathbf{3 2 0} \mathbf{\text { mTorr }}$} & \multicolumn{2}{c|}{$\mathbf{p}=\mathbf{1 0 0} \mathbf{\text { TTorr }}$} \\
\hline $\mathbf{B}_{\mathbf{z}}^{\mathbf{0}}$ & $\mathbf{0}$ & $\mathbf{2 . 9} \mathbf{k G}$ & $\mathbf{0}$ & $\mathbf{2 . 9} \mathbf{k G}$ \\
\hline $\mathbf{N}_{\mathbf{e}}^{\mathbf{0}}, \mathbf{1 0}^{\mathbf{1 6}} \mathbf{c m}^{-\mathbf{3}}$ & $0.9 \pm 0.1$ & $1.3 \pm 0.3$ & $0.3 \pm 0.1$ & $0.4 \pm 0.1$ \\
\hline $\mathbf{N}_{\mathbf{e}}^{\mathbf{x}}, \mathbf{1 0}^{\mathbf{1 6}} \mathbf{c m}^{-\mathbf{3}}$ & $3.4 \pm 0.3$ & $1.1 \pm 0.2$ & $18.0 \pm 2.0$ & $2.5 \pm 0.9$ \\
\hline $\mathbf{T}_{\mathbf{i}}, \mathbf{e V}$ & $50 \pm 5$ & $50 \pm 5$ & $50 \pm 8$ & $40 \pm 10$ \\
\hline $\mathbf{W}_{\mathbf{x}}, \mathbf{e V}$ & $400 \pm 80$ & 0 & $1300 \pm 300$ & $100 \pm 40$ \\
\hline
\end{tabular}

\section{Conclusions}

1. An appreciable difference has been found experimentally between broadenings of the He I and He II lines measured simultaneously in two mutually perpendicular directions ( $x$ and $z$ axes) in the CSs.

2. It was shown that this spectral effect was caused by superthermal plasma jets directed along the sheet width ( $x$-axis). The tangential jets' energy is in the range $W x=400-1300 \mathrm{eV}$ while the initial pressure is in the range $p=320-100 \mathrm{mTorr}$. Helium atoms also accelerate along the sheet width ( $x$-axis) to superthermal velocities, probably, through charge-exchange process.

\section{References}

Ackermann, U., Finken, K.H., \& Musielok, J., 1985, Phys. Rev. A., 31, 2597

Barnard, A.J., Cooper, J., \& Shamey, L.J., 1969, A\&A, 1, 28

Frank, A.G., 2010, UFN, 180, 982

Frank, A.G., 2012, EAS Publ. Ser., 58, 57

Kyrie, N.P., Markov, V.S., \& Frank, A.G., 2010, Plasma Phys. Rep., 36, 357

Pittman, T.L., \& Fleurier, C., 1986, Phys. Rev. A., 31, 1291

Priest E.R., \& Forbes, T., 2000, Magnetic reconnection. MHD theory and applications

(Cambridge University Press)

Syrovatskii, S.I., 1981, ARA\&A, 19, 163 